\title{
Preliminary study on the virulence of XDR-TB: Low virulence owing to less cytokine expression through the TLR 2 and TLR4 pathways in BLAB/C mice ${ }^{1}$
}

\author{
Zhaogang Sun ${ }^{\mathrm{a}, \mathrm{c}}$, Weicong Ren ${ }^{\mathrm{a}, \mathrm{c}}$, Yuhui $\mathrm{Xu}^{\mathrm{a}, \mathrm{c}}$, Haiqing Zhang ${ }^{\mathrm{b}, \mathrm{c}}$ and Chuanyou $\mathrm{Li}^{\mathrm{a}, \mathrm{c}, *}$ \\ ${ }^{a}$ Beijing Key Laboratory on Drug Resistance in Tuberculosis Research, Beijing 101149, China \\ ${ }^{b}$ Department of Pathology, Beijing Tuberculosis \& Thoracic Tumor Research Institute, Beijing \\ 101149, China \\ ${ }^{c}$ Beijing Chest Hospital, Capital Medical University, Beijing 101149, China
}

\begin{abstract}
Extensively drug-resistant tuberculosis (XDR-TB) imposes a new threat to the world. In this study, XDR-TB and drug-sensitive TB (DS-TB) strains with similar genotypes were investigated. The results showed that BLAB/C mice infected with XDR-TB had reduced Toll-like receptor (TLR) 2 and TLR4 expression in lung tissues than DS-TB mice at 2 weeks post-infection. Mice infected with XDR-TB showed lower levels of TNF- $\alpha$, IFN- $\gamma$, IL- 4 and IL-10 in the sera than those infected with DS-TB. Moreover, mice infected with XDR-TB survived longer period and had less severe alveolar damage and smaller granulomas than those infected with DS-TB. These results were further confirmed in THP-1 cells. Using the flow cytometry and qRT-PCR methods, we found that XDR-TB stimulated lower TLR2 and TLR4 expression. Based on the above results, we cautiously inferred that low virulence of XDR-TB owed to the less cytokine expression through the TLR 2 and 4 pathways. The effect of XDR-TB and DS-TB can be further tested in TLR2 or TLR4 knock-out mice and TLRs-silenced THP-1 cells.
\end{abstract}

Keywords: Extensively drug-resistant tuberculosis, toll-like receptor, cytokine, virulence

\section{Introduction}

XDR-TB imposes new threats to global health. According to WHO's report, 84 countries, including China, had confirmed at least one case of XDR-TB by October 2012 [1]. Treatment for XDR-TB has been tried by several countries, but few promising outcomes were gained [2-4]. Compared with patients with MDR-TB, patients with XDR-TB show increased mortality and have longer hospitalization, treatment duration and conversion time [2-4]. As XDR-TB treatment is a complicate process, it is necessary to investigate the virulence of special microbes of XDR-TB.

\footnotetext{
${ }^{1}$ All authors disclose no conflicts of interest.

*Corresponding author: Chuanyou Li, Beijing Key Laboratory on Drug Resistance in Tuberculosis Research, Beijing Chest Hospital, Capital Medical University, Beijing 101149, China. Tel.: 86-10-69545354; Fax: 86-10-69546819; E-mail: lichuanyou6688@hotmail.com.
} 
TLRs have emerged as key players in the induction of anti-microbial immune responses by triggering the anti-microbial innate immune response and shaping the adaptive immune response [5-7]. It has been reported that TLRs, especially TLR2 and TLR4, plays an important role in activating the immune response by M. tuberculosis. M. tuberculosis-induced TNF- $\alpha$ production is found to be primarily TLR2-dependent, but other TLRs contribute to proinflammatory signaling [8-10]. Previously study suggests that macrophage activation and clearance of mycobacteria are depended on TLR4 [11], but M. tuberculosis 19-kd protein mediates the inhibition of MHC class II expression and antigen processing through TLR2, which may allow intracellular bacilli to evade immune surveillance and thereby promote chronic infection [12]. The virtue of XDR-TB chronic infection is reported in Peru. XDR-TB patients showed longer cumulative months of previous treatment (34.7 vs. 25.1 months, $\mathrm{p}<0.001)$ and duration of treatment ( 26.0 vs. 24.8 months, $\mathrm{p}=0.33)$, but no significant difference in poor outcome and death than MDR-TB patients [4].

As TLRs play such critical roles in infection and pathogenesis, in this study, we investigated the expression of major TLRs, TLR2 and TLR4, and cytokines in THP-1 cells and in BLAB/c mice infected either by XDR-TB or DS-TB. It is a preliminary study on the virulence of XDR-TB.

\section{Materials and methods}

\subsection{Abbreviations/nomenclature}

XDR-TB, extensively drug-resistant tuberculosis; TLRs, Toll-like receptors; DS-TB, drug-sensitive tuberculosis; TNF- $\alpha$, tumor necrosis factor; IFN- $\gamma$, interferon $\gamma$; IL-4, interleukin 4; IL-10, interleukin 10; OADC, oleic acid-albumin-dextrose-catalase; FCS, fetal calf serum; PMA, Phorbol-12-myristate-13-acetate; MOI, multiplicity of infection; CFU, colony forming units; LAM, lipoarabinomannan; PIM, mannosylated phosphotidylinositol.

\subsection{Strains and cell culture}

In the present study, we selected two DS-TB strains (A36 and A25) and two XDR-TB strains (B42 and E19) [13] as typical clinical isolates. These strains carry identical Beijing genotypes and were cultivated first on L-J slants, and then inoculated in Middle Brook 7H9 medium plus 10\% OADC (BD Co., MD, USA). After ten days, the cultures of logarithmic-phase bacilli were harvested and ultrasonicated twice $(20 \mathrm{~W}, 10 \mathrm{~s})$. The supernatant concentration of bacteria was determined by McFar Land after putting it aside for $10 \mathrm{~min}$. The mycobacteria were pelleted by centrifugation at 5,000 x g for $10 \mathrm{~min}$ and washed twice with fresh medium.

THP-1 cells were grown in RPMI 1640 medium (2 mM L-glutamine) supplemented with $10 \%$ FCS at $37^{\circ} \mathrm{C}$ in a $5 \% \mathrm{CO} 2$ incubator. Medium was changed every three days. For each experiment, cells were individually plated at density of $5 \times 10^{5}$ cells/well in twelve-well plates and treated with 5ng/mL PMA (Sigma-Aldrich, St. Louis, MO) in RPMI 1640/10\% FCS for 24 hours [14]. The adherent cells were washed at least 3 times with PBS. Before addition of M. tuberculosis, PMA-containing medium was removed from the adherent THP-1 cells, and was replaced with $1.5 \mathrm{ml}$ of RPMI 1640/5\% FBS medium containing M. tuberculosis at a multiplicity of infection of 1-2 (MOI). After incubated for $4 \mathrm{~h}$ at $37^{\circ} \mathrm{C}$, extracellular bacteria were removed by washing three times with RPMI 1640, and THP-1 cells were cultured in fresh RPMI 1640 with $5 \%$ FCS medium at $37^{\circ} \mathrm{C}$ until the time of harvest. 


\subsection{Mice infected with bacteria and histological techniques}

Ethical committee of Beijing TB \& thoracic Tumor Research Institute gave the ethical clearance. 8-week-old female BALB/c mice (18-20 g, Laboratory Animal Center of Chinese Academy of Medical Science, China) were infected by injecting A36, A25, B42 or E19 cells into the caudal vein. All mice were maintained in an animal biosafety facility. The study protocol was approved by the ethical committee of Beijing Tuberculosis \& Thoracic Tumor Research Institute, Beijing. Lung or spleen tissues were partially used to determine CFU, while other parts were used for histopathology. The histological analysis of lung and spleen was performed using the organs harvested 4 and 8 weeks post-infection. Lungs were fixed in 10\% formalin and embedded in paraffin using standard techniques. Sections $(5 \mu \mathrm{m}$ thick) were stained with hematoxilin and eosin and subsequently reviewed by a pathologist for histology. Lungs or spleens from at least 3 mice in each group were analyzed.

\subsection{Enzyme linked immunoabsorbant assay (ELISA)}

At the specific times of 2 and 4 weeks post infection, the mouse sera were collected and assayed for cytokine production using an ELISA kit (Jingmei Biotech., Beijing, China). The levels of IFN- $\gamma$, TNF- $\alpha$, IL-4, and IL-10 were determined according to the manufacturer's instructions.

\subsection{Flow cytometry}

THP-1 cells were infected at an MOI of 1-2 for 4 hours as previously described [15] and collected at 24 hours post infection. $5 \times 10^{5}$ cells were washed with $1 \mathrm{ml}$ PBS and resuspended in $100 \mu \mathrm{l}$ of PBS containing 2\% FCS. 1/2 cells were incubated with an FITC-labeled human TLR2 and 1/2 cells were incubated with PE- labeled human TLR4. The cells were fixed with $2 \%$ paraformaldehyde for 1 hour and analyzed by flow cytometry (FACSCalibur, BD Biosciences, NJ, USA)

\subsection{RNA isolation and $q R T-P C R$}

RNA was collected using an RNeasy kit (Biotake, China) for preparation of RNA from adherent mammalian cells according to the manufacturer's instructions. RNA yield was determined by a Thermo Scientific NanoDrop 2000c. A total of $2 \mu \mathrm{g}$ RNA was used in a $20 \mu \mathrm{l}$ reverse transcription reaction and transcribed using a RT kit (TransGen Biotech) according to the manufacturer's instructions with oligo-dT (Ambion, Austin, TX) at a final concentration of $5 \mu \mathrm{M}$ and RNase inhibitor (Promega) at a final concentration of $0.5 \mathrm{U} / \mu \mathrm{l}$. Reverse transcription was conducted at $42^{\circ} \mathrm{C}$ for $30 \mathrm{~min}$, followed by heat inactivation at $85^{\circ} \mathrm{C}$ for $5 \mathrm{~min}$. cDNA was $1: 4$ diluted and $1 \mu \mathrm{l}$ was used as template for quantitative real-time PCR (qRT-PCR). PCR was performed in a volume of $15 \mu \mathrm{l}$ in an ABI PRISM 5700HT sequence detection system (Perkin-Elmer). Negative control reactions were subjected to the same qRT-PCR reaction. Human $\beta$-actin was used as the control gene for quantification. The primer sequences were 5' -TGT TTG GCG TCG AAT GTG A -3' and 5' AGG ACT TTA TCG CAG CTC TCA GA -3' for TLR2; 5' -GATTGCTCAGACCTGGCAGTT -3' and 5' -TGT CCT CCC ACT CCA GGT AAG T -3' for TLR4; and 5' -TGACGTGGACATCCGCAAAG -3' and 5'CTGGAAGGTGGACAGCGAGG- 3 ' for inner control of $\beta$-actin. 


\subsection{Western blot}

Lung tissues ( 2 and 4 weeks post-infection) were homogenized with homogenizer in lysis buffer (Tris-HCL-300ul per 5mg of tissue) plus protease inhibitor cocktail (Sigma alrich). A Bradford protein assay kit (Pierce, USA) was used to measure protein concentration. We used SDS-PAGE to separate proteins. TLR2 and TLR4 were detected using specific antibodies (rabbit anti-mouse TLR2 and TLR4, rabbit anti-mouse $\beta$-actin andgoat anti-rabbit IgG-HRP antibodies from Biolegend, San diego, CA, USA) and ECL detection system (Pierce, USA). Appropriate antibody controls were used for each experiment and no signal was observed. The densitometric ratios were determined as reported earlier by Gel-Pro Analyzer (Media Cybernetics, USA) [16].

\section{Results}

\subsection{Animal death and histological analysis of lung and spleen tissues of infected BALB/c mice}

In this study, four strains with identical Beijing genotypes were used for comparison between XDR-TB and DS-TB (Table 1). Within 16 weeks, eleven BALB/c mice infected with $10^{5} \mathrm{CFU}$ of DS-TB A36 or A25 cells were all dead, while none of BALB/c mice infected with the same dose of XDR-TB B42 or E19 cells died during the first 10 weeks. The Median survival days for the mice infected with XDR-TB (10 CFU) and DS-TB were 155 (138.3-176.3, $25 \%$-75\% percentile) and 54 (47.3-75.0, $25 \%-75 \%$ percentile), individually (Figure 1A).

The bacilli burden of lung and spleen was not significantly different between XDR-TB and DR-TB mice at all the checked time points. Compared to XDR-TB mice, the mice infected with DS-TB had worse histopathology, of spleen and lung showing more severe alveolar damage and bigger granulomas (Figures 1B and 1C).

Table 1

Genotype and drug resistance patterns of drug-sensitive strains (A36 and A25) and extensively drug-resistant strains (B42and E19) used in this study

\begin{tabular}{|c|c|c|c|c|c|}
\hline \multicolumn{2}{|l|}{ strain } & A36 & A25 & B42 & E19 \\
\hline \multicolumn{2}{|c|}{ Spoligotyping pattern } & 000000000003771 & 000000000003771 & 000000000003771 & 000000000003771 \\
\hline \multicolumn{2}{|c|}{ MIRU pattern } & 223325163533 & 223325163533 & 223325163533 & 223325163533 \\
\hline \multicolumn{2}{|c|}{ VNTR pattern } & 4243586 & 4243585 & 4243586 & 4243516 \\
\hline \multirow{4}{*}{ Drug } & $\mathrm{INH}(1,10)^{*}$ & 0.25 & 0.125 & 20 & 20 \\
\hline & $\operatorname{RIF}(50,250)$ & 12.5 & 12.5 & 500 & 2000 \\
\hline & $\operatorname{OFX}(5,50)$ & 1.25 & 1.25 & 10 & 40 \\
\hline & $\operatorname{AmK}(5,50)$ & 0.625 & 1.25 & 80 & 160 \\
\hline \multirow{2}{*}{ resistance } & $\mathrm{SM}(10,100)$ & 2.5 & 2.5 & 1600 & 20 \\
\hline & $\operatorname{PZA}(50,250)$ & 12.5 & 6.25 & 800 & 3200 \\
\hline \multirow{3}{*}{ pattern } & $\operatorname{EMB}(5,50)$ & 1.25 & 1.25 & 20 & 10 \\
\hline & $\operatorname{PAS}(1,10)$ & 0.125 & 0.25 & 4 & 32 \\
\hline & $\overline{\mathrm{ETH}}(25,100)$ & 3.125 & 3.125 & 50 & 50 \\
\hline
\end{tabular}

Note: ${ }^{*}$, drug-concentration standards for low or high drug resistance, $\mathrm{g} / \mathrm{ml}$.

MIRU, mycobacterial interspersed repetitive units. VNTR, variable number tandem repeats.INH, isoniazid; RIF, rifampin; OFX, ofloxacin; AmK, amikacin; SM, streptomycin; PZA, pyrazinamide; EMB, ethambutol; PAS, p-aminosalicylic acid sodium; ETH, ethionamide. 


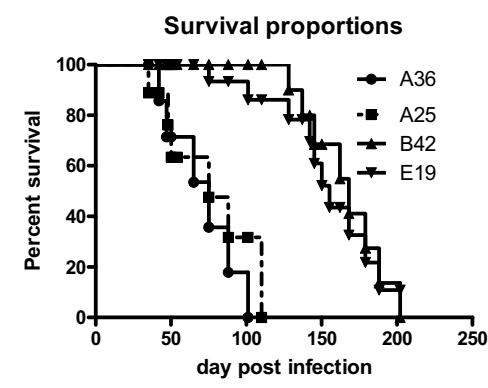

A

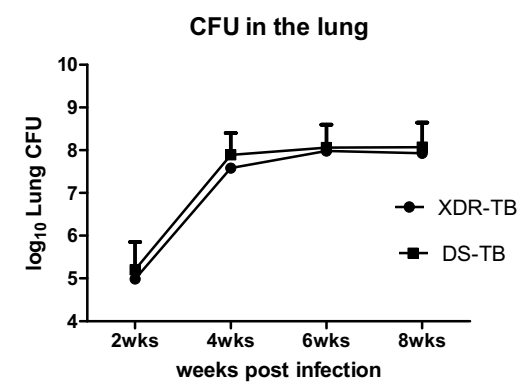

B1

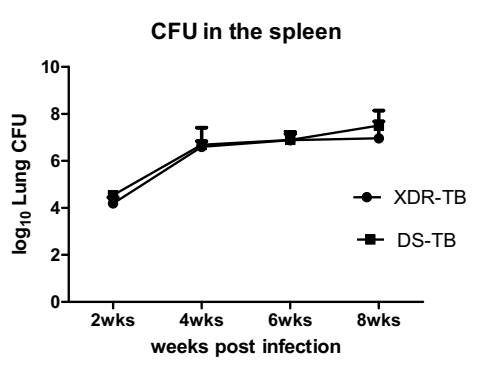

B2

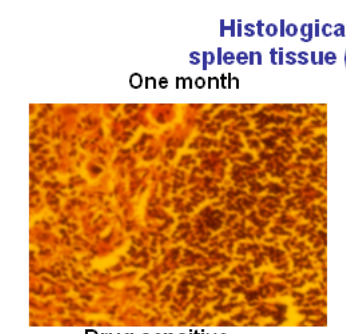

Drug sensitive

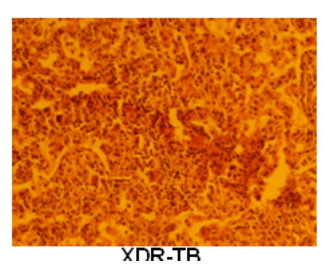

Techniques E. method)

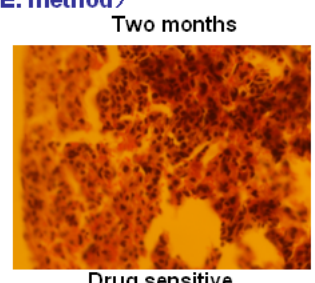

Drug sensitive

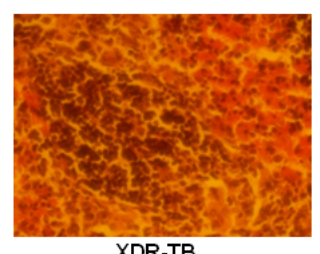

XNR-TR
Histological Techniques lung tissue (H. E. method)

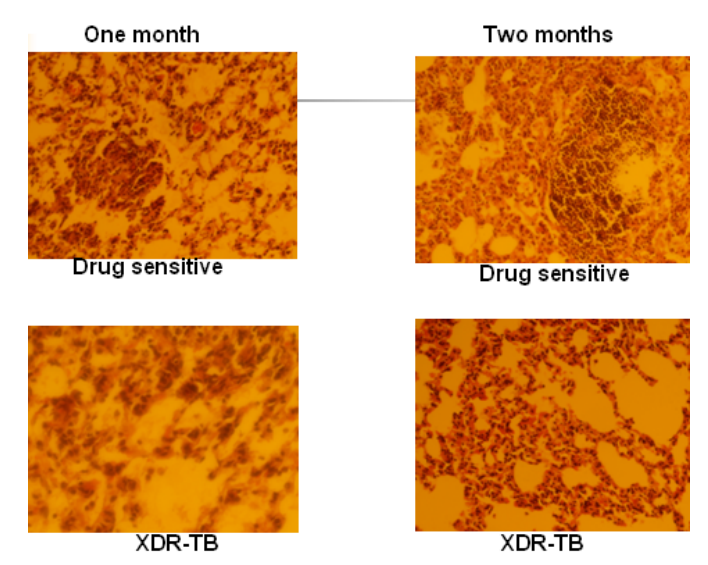

C

Fig. 1. Survival, bacterial burden and pathology damage in spleen and lung of BALB/c mice infected with $10^{5} \mathrm{CFU}$ of $M$. tuberculosis. A, the death curve of the mice infected with the B42, E19, A36 and A25 cells; B, the bacterial burden of lung (left) and spleen (right) of infected mice at individual time points; C, Histological analysis of lung and spleen after 4 and 8 weeks infection with either XDR-TB or DS-TB. Tissue sections from mice infected with a low dose (10 CFU) of $M$. tuberculosis were formalin-fixed, paraffin-embedded and stained with hematoxylin and eosin at 4 and 8 weeks post-infection.

\subsection{ELISA analysis of serum cytokine levels in infected mice}

In order to better understand the mechanism of pathogenesis, which is reflected in the different immune responses to the DS-TB and XDR-TB strains, we measured serum levels of TNF- $\alpha$, IFN- $\gamma$, IL-4, and IL-10. Figure 2 shows the mice infected with DS-TB strains secreted more TNF- $\alpha$ (Figure 2A), IL-4 (Figure 2B) and IL-10 (Figure 2C) at 2 weeks and 4weeks post-infection, almost doubled for the detected cytokines in the mice infected with XDR-TB strains.

\subsection{Detect TLR2 and TLR4 expression in THP-1 cells at different time points by flow cytometer and $q R T-P C R$}

To verify the different expression of TLR2 and TLR4 caused by XDR-TB and DS-TB infection in $\mathrm{BALB} / \mathrm{c}$ mice, we further tested the expression levels in activated THP-1 cells. The flow cytometry results showed that XDR-TB induced less expression of TLR2 and TLR4 (with the average of $4.88 \%$ and $0.65 \%$, respectively) than DS-TB did (with the average of $7.11 \%$ and $0.88 \%$, respectively) after 

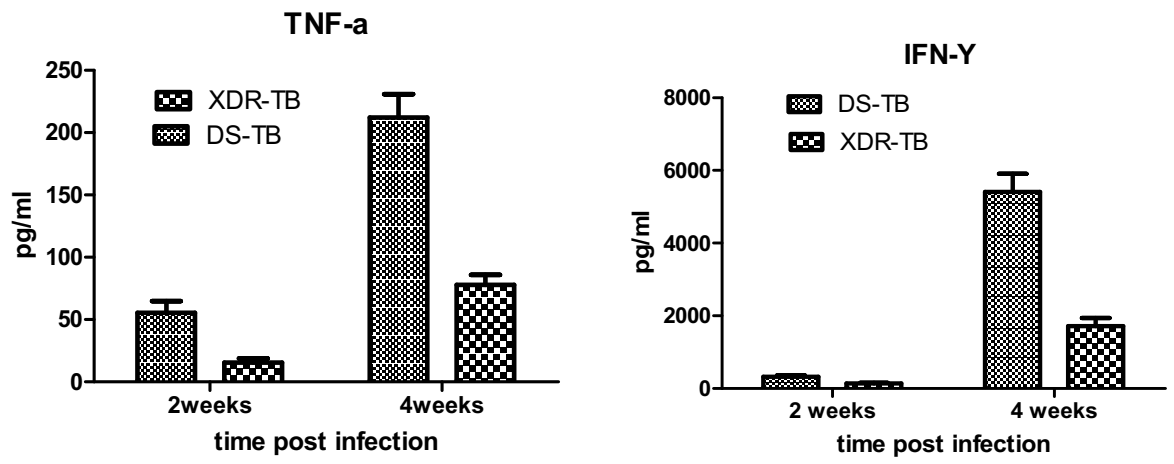

IL-4

$$
\text { IL-10 }
$$
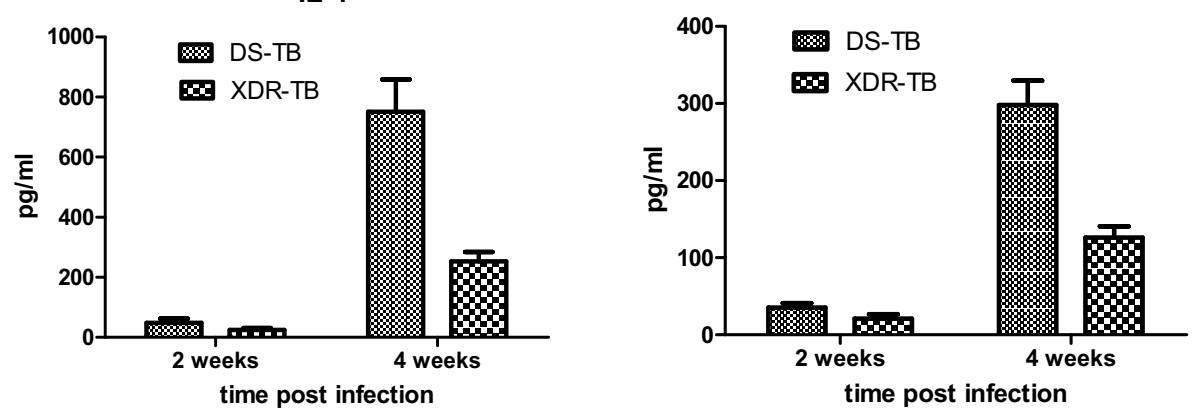

Fig. 2. Serum cytokine levels of BALB/c mice infected with XDR-TB or DS-TB strains, respectively. A, TNF- $\alpha$; B, IFN- $\gamma$; C, IL-4 and D, IL-10. (pg/ml).

infection for 24h (Figures 3A and 3B). It was confirmed by qRT-PCR (Figures 3C and 3D). qRT-PCR results also indicated that cells infected with DS-TB expressed more, but not significantly more, TLR2 and TLR4 compared to those infected with XDR-TB at $12 \mathrm{~h}, 24 \mathrm{~h}$ and $48 \mathrm{~h}$ post-infection (Figures $3 \mathrm{C}$ and $3 \mathrm{D})$.

\subsection{Western Blot assays for TLR2 and TLR4 expression}

To profile expression of TLR2 and TLR4, western blotting was performed to test lung proteins at 2 and 4 weeks post-infection of XDR-TB or DS-TB and then the images were digitized to get densitometric ratios. TLR4 was more expressed than TLR2 at both time points and both TLR4 and TLR2 had increased expression at 4 weeks post-infection versus 2 weeks post-infection. More importantly, at 2 weeks post-infection both TLR2 and TLR 4 expressed less in lung tissues of the mice infected with XDR-TB than those infected with DS-TB (Figure 4). 
TLR2
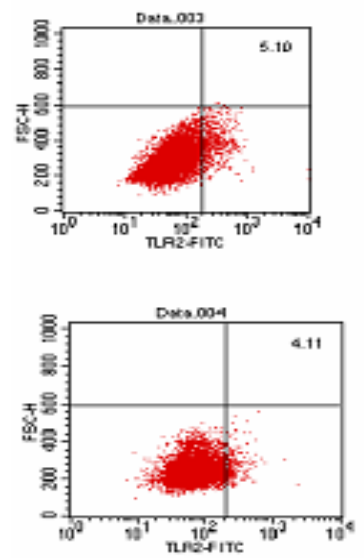

A
TLR4
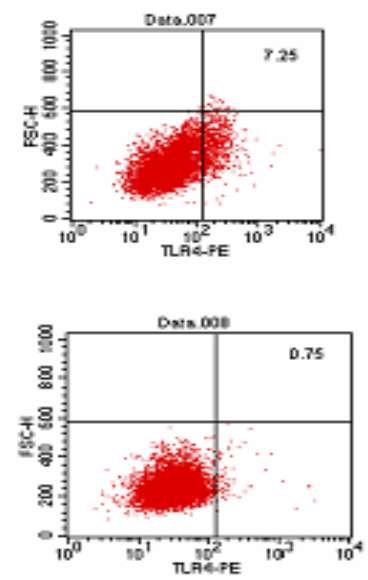

$\mathbf{B}$
TLR2

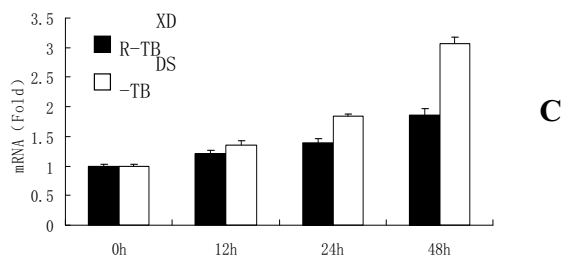

TLR4

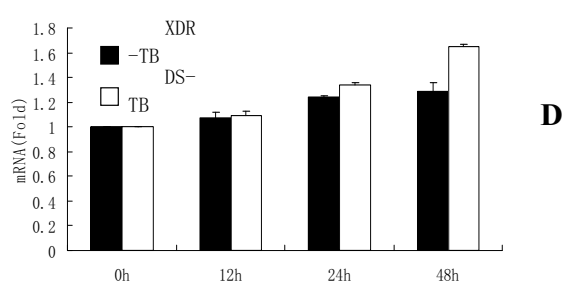

Fig. 3. Expression profile of TLR2 and TLR4, and cell apoptosis analysis by flow cytometry after infection with DS-TB (A36 and A25) or XDR-TB (B42 and E19). A and B: Flow Cytometry analysis of TLR2 and TLR4 expression in THP-1 cells infected with DS-TB (upper) or XDR-TB (lower). C and D: Transcription of TLR2 and TLR4 in THP-1 cells. They are quantified by real-time PCR after 12, 24 and 48 hours infection with DS-TB or XDR-TB. The most representative result of three repeats from all the experiments is shown in this figure.

\section{XDR-TB DS-TB XDR-TB DS-TB}

$\begin{array}{ll}0.20 \pm 0.14 & 0.31 \pm 0.22 * \\ 0.27 \pm 0.18 & 0.42 \pm 0.19 *\end{array}$

Dentometric ratio

XDR-TB DS-TB

$0.51 \pm 0.37 \quad 0.48 \pm 0.20$

$066 \pm 0.31 \quad 0.73 \pm 0.29$

Dentometric ratio
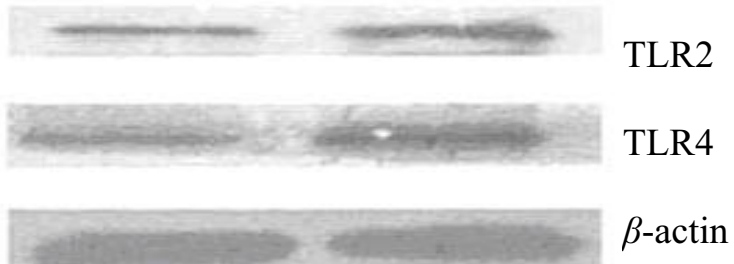

A $\beta$-actin

DS-TB

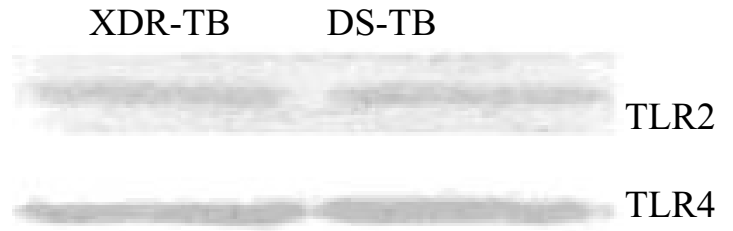

$\beta$-actin

$\mathrm{B}$

Fig. 4. Representative western blotting results depicting the TLR2 and TLR4 protein expression in BALB/c mice infected with DR-TB or XDR-TB strains. At 2 (A) and 4 weeks (B) post-infection, lung tissues were collected, lysed, and $20 \mu \mathrm{g}$ protein was blotted for the indicated proteins and $\beta$-actin as described in Materials and methods. The densitometric ratios are indicated as described in Materials and methods. 


\section{Discussion}

It is well known that TLRs play very important roles in the induction of anti-TB immune responses. By comparing with DS-TB, this study demonstrated that XDR-TB exhibited less virulence through the TLRs-cytokines signal pathway. For the safety reason, our study picked only one or two XDR-TB strains, which have almost similar genotypes as the DS-TB strains, for our investigation on the virulence. This is the preliminary study on virulence of XDR-TB.

A different expression level of TLRs is associated with the gravity of lesion in some diseases. A previous report on the lesion in patients with self-healing tuberculoid form shows that TLR2 and TLR1 expression in skin lesions is much more than in largely disseminated lepromatous patients [17]. In this study, TLR2 and TLR4 were also differently expressed between mice infected with XDR-TB and DS-TB strains. XDR-TB induced less expression of TLR2 and TLR4 than DR-TB did (Figure 2). By applying the TLRs inhibitor in THP-1 cells, our study confirmed that the differences in TLR expression were attributed to the different secretion levels of cytokine. It means that XDR-TB induces much weaker active immuno-responses for MTB being destroyed. TLRs mediate cellular activation through components of mycobacteria, of which TLR2 and TLR4 are the two most important TLRs activated by the major immuno-stimulatory components, such as $19-\mathrm{kDa}$ lipoprotein $[9,18]$, LAM [19] and PIM [20]. It is likely that some changes happened in XDR-TB as described in a recent report on the development of antibiotic resistance in a monoclonal MTB-infected patient [17]. The antibiotic resistance of XDR-TB results in a physiological burden, or fitness cost, that is associated with mutations in cell wall synthesis genes [21,22]. It may aid in interpreting our results, which showed lower levels of cytokines in XDR-TB infected BALB/c mice compared with DS-TB infected mice.

Following TLR activation, the MyD88-dependent and -independent signaling pathways are triggered, leading to the nuclear translocation of NF-kB, a transcription factor involved in modulating many immune response genes, such as Type- 1 and Type 2 cytokines [17,23,24]. Combined with the facts that expression of TLR2 and TLR4 decreased in XDR-TB infected THP-1 cells and BLAB/c mice in this study, we propose that decreased expression of TLR2 and TLR4 causes reduced expression of cytokines, including type- 1 and type- 2 cytokines.

Tissue damage may also result from TLR-induced cytokine secretion and apoptosis in response to mycobacteria [25-27]. In this study, the bacterial burden in spleen and lung of the BALB/c mice infected with XDR-TB was almost same as that in DS-TB mice at two months post-infection (Figure 4). It suggests that the bacterial burden is not the main reason of mouse death. The possible reason is that the acute immune response may cause much greater damage to hosts infected with DS-TB than with XDR-TB.

In conclusion, TLR-2 and TLR-4 play important roles in TB infections and disease progress. When TB strains develop drug resistance, changing from drug sensitive to extensively drug resistant cells, they modify their capabilities to activate TLR-2 and TLR-4 for the natural protection by the host immo-systems. This study is a preliminary report on the effect of the evolution of drug resistance on bacterial survival in hosts. However, more TB cases and bacterial molecular evidences are needed to confirm these changes in bacteria. Future work should focus on the effect of XDR-TB and DS-TB infection onTLR2 or TLR4 knock-out mice and TLRs-silenced THP-1 cells. 


\section{Acknowledgement}

The authors are grateful to Dr. B. Chen in Albert Einstein College of Medicine for critical review of the manuscript. This work was supported by the Key Project Specialized for Infectious Diseases of the Chinese Ministry of Health (2013ZX10003009) the National Natural Science Foundation of China (30901283) and Beijing Municipal senior personnel of health training program (2011-3-069).

\section{References}

[1] World Health Organization (WHO), Global Tuberculosis Report 2012, http://apps.who.int/iris/bitstream/10665/75938/1/9789241564502_eng.pdf. (2013)

[2] H.-R. Kim, S.S. Hwang, H.J. Kim, S.M. Lee, C.G. Yoo, Y.W. Kim, S.K. Han, Y.S. Shim and J.J. Yim, Impact of extensive drug resistance on treatment outcomes in non-HIV-infected patients with multidrug-resistant tuberculosis, Clin. Infect. Dis. 45 (2007), 1290-1295.

[3] G.B. Migliori, J. Ortmann, E. Girardi, G. Besozzi, C. Lange, D.M. Cirillo et al., Extensively drug-resistant tuberculosis, Italy and Germany, Emerg. Infect. Dis. 13 (2007), 780-781.

[4] C.D. Mitnick, S.S. Shin, K.J. Seung, M.L. Rich, S.S. Atwood, J.J. Furin et al., Comprehensive treatment of extensively drug-resistant tuberculosis, N. Engl. J. Med. 359 (2008), 563-574.

[5] C. Li, H. Li, K. Jiang, J. Li and X. Gai, TLR4 signaling pathway in mouse lewis lung cancer cells promotes the expression of TGF- $\beta 1$ and IL-10 and tumor cells migration, Bio-Med. Mater. Eng. 24 (2014), 869-875.

[6] B. Salaun, P. Romero and S. Lebecque, Toll-like receptors' two-edged sword: when immunity meets apoptosis, Eur. J. Immunol. 37 (2007), 3311-3318.

[7] D. Xu, H. Liu and M. Komai-Koma, Direct and indirect role of Toll-like receptors in T cell mediated immunity, Cell Mol. Immuno. 1 (2004), 239-246.

[8] K. Takeda, T. Kaisho and S. Akir, Toll-like receptors, Annu. Rev. Immunol. 21 (2003), 335-376.

[9] S. Thoma-Uszynski, S. Stenger, O. Takeuchi, M.T. Ochoa, M. Engele, P.A. Sieling et al., Induction of direct antimicrobial activity through mammalian toll-like receptors, Science 291 (2001), 1544-1547.

[10] D.M. Underhill, A. Ozinsky, K.D. Smit and A. Aderem, Toll-like receptor-2 mediates mycobacteria-induced proinflammatory signaling in macrophages, Proc. Natl. Acad. Sci. 96 (1999), 14459-14463.

[11] B. Abel, N. Thieblemont, V.J. Quesniaux, N. Brown, J. Mpagi, K. Miyake et al., Toll-like receptor 4 expression is required to control chronic mycobacterium tuberculosis infection in mice, J. Immuno. 169 (2002), 3155-3162.

[12] E. H. Noss, R.K. Pai, T.J. Sellati, J.D. Radolf, J. Belisle, D.T. Golenbock et al., Toll-like receptor 2-dependent inhibition of macrophage class II MHC expression and antigen processing by $19-\mathrm{kDa}$ lipoprotein of Mycobacterium tuberculosis, $\mathrm{J}$. Immunol. 167 (2001), 910-918.

[13] Z. Sun, Y. Chao, X. Zhang, J. Zhang, Y. Li, Y. Qiu et al., Characterizaton of extensively drug-resistant mycobacterium tuberculosis clinical isolates in China, J. Clin. Microb. 46 (2008), 4075-4077.

[14] M.N. Chahine, J. Deniset, E. Dibrov, S. Hirono, D.P. Blackwoo, J.A. Austri et al., Oxidized LDL promotes the mitogenic actions of Chlamydia pneumoniae in vascular smooth muscle, Cardiovasc. Res. 92 (2011), 476-483.

[15] J. Chan, Y. Xing, R.S. Magliozzo and B.R. Bloom, Killing of virulent Mycobacterium tuberculosis by reactive nitrogen intermediates produced by activated murine macrophages, J. Exp. Med. 175 (1992), 1111-1122.

[16] M.R. Dasu, S. Devaraj, L. Zhao, D.H. Hwang and J.I. ialal, High glucose induces toll-like receptor expression in human monocytes: Mechanism of activation, Diabetes 57 (2008), 3090-3098.

[17] C. Holscher, R.A. Atkinson, B. Arendse, N. Brown, E. Myburgh, G. Alber, F. Brombacher et al., A protective and agonistic function of IL-12p40 in mycobacterial infection, J. Immunol. 167 (2001), 6957-6966.

[18] C.J. Hertz, S.M. Kiertscher, P.J. Godowski, D.A. Bouis, M.V. Norgard, M.D. Roth et al., Microbial lipopeptides stimulate dendritic cell maturationvia Toll-like receptor 2, J. Immunol. 166 (2001), 2444-2450.

[19] T.K. Means, S. Wang, E. Lien, A. Yoshimura, D.T. Golenbock and M.J. Fenton, Human toll-like receptors mediate cellular activation by Mycobacterium tuberculosis, J. Immunol. 163 (1999), 3920-3927.

[20] B.W. Jones, T.K. Means, K.A. Heldwein, M.A. Keen, P.J. Hill, J.T. Belisle et al., Different toll-like receptor agonists induce distinct macrophage responses, J. Leukoc. Biol. 69 (2001), 1036-1044.

[21] A. Davies, Comparison of fitness of two isolates of Mycobacterium tuberculosis, one of which had developed multi-drug resistance during the course of treatment, J. Infect. 41 (2003), 184-187. 
[22] S.H. Maria, J. Werngren, J. Aronsson, S. Hoffner and D.I. Andersson, Dynamics of antibiotic resistant Mycobacterium tuberculosis during long-term infection and antibiotic treatment, PLoS One 6 (2011), e21147.

[23] M.B. Reed, P. Domenech, C. Manca, H. Su, A.K. Barczak, B.N. Kreiswirth et al., A glycolipid of hypervirulent tuberculosis strains that inhibits the innateimmune response, Nature 431 (2004), 84-87.

[24] Y-P Li, V. Latger-Canard, L. Marchal, N. Li, J-P. O and J-F. Stoltz, The regulatory role of dendritic cells in the immune tolerance, Bio-Med. Mater. Eng. 16 (2006), S163-S170.

[25] M.O. Hernandez, I. Neves, J.S. Sales, D.S. Carvalho, E.N. Sarno and E.P. Sampaio, Induction of apoptosis in monocytes by Mycobacterium leprae in vitro: A possible role for tumour necrosis factor-alpha, Immunology 109 (2003), 156-164.

[26] K.S. Michelsen, A. Aicher, M. Mohaupt, T. Hartung, S. Dimmeler, C.J. Kirschning et al., The role of toll-like receptors (TLRs) in bacteria-induced maturation of murine dendritic cells (DCS), peptidoglycan and lipoteichoic acid are inducers of DC maturation and require TLR2, J. Biol. Chem. 276 (2001), 25680-25686.

[27] K.A. Zarember and P.J. Godowski, Tissue expression of human toll-like receptors and differential regulation of toll-like receptor mRNAs in leukocytes in response to microbes, their products, and cytokines, J. Immunol. 168 (2002), 554-561. 